tives. Scanning a TEM negative with this compensation applied causes severe posterization, i.e., loss of gray levels, in the 8-bit output image. The posterization effect might not be obvious in grayscale histograms taken over the entire scanned image, but will appear as a stairstep effect in linescan profiles taken across high-contrast image features.

To avoid posterization in scanning negatives, try the positive transparency scanning mode. The image contrast can be inverted with settings in the scanner control program, or during later manipulations with Photoshop. The scanner software may still apply built-in contrast compensation, but it won't be as severe as that in the negative transparency mode. Unfortunately, most scanner drivers provide no (or limited) user control over the built-in film contrast compensation. Microtek's ScanWizard program, for one, contains an option to force linear gamma in the positive transparency mode but not in the negative transparency mode. I sometimes find the forced linear gamma mode useful for very high-contrast micrographs.

This tip might help, but it won't teach how to produce optimum scans. For that, I highly recommend the book Real World Photoshop 4 by David Blatner and Bruce Fraser, Peachpit Press, 1997

Larry Thomas, Washington State University

1) Put a small drop of concentrated sulfuric acid on the mounted epon section.

2) Count to 10 slowly, then rinse it off under the tap.

3) Put a drop of any basic dye on the section. Toluidin blue is as good as any, but neutral red, safranin, basic Fuchsin, etc. are OK too. I like toluidine blue best. It doesn't need to be an alkaline solution; any $\mathrm{pH}$ is OK.

4) Wait for about a minute, rinse under the tap and thoroughly air-dry

5) Optionally treat with $100 \%$ alcohol (this removes a little of the stain: not usually necessary), followed by xylene and a resinous mounting medium.

Some people prefer to mount in epon, which takes longer but gives better transparency. Others don't mount at all, but examine directly in immersion oil, as for a blood smear.

The sulfuric acid pretreatment (a traditional histochemical tick, but destructive to non-plastic sections) changes hydroxyl groups and some amines to half-sulfate esters or sulphoamino groups, both of which ionize as strong acids. Consequently, a basic dye at any $\mathrm{pH}$ will bind electrostatically to pretty well everything in the tissue, and you get metachromatic effects too: purples and reds with blue dyes, orange with red dye

A reference for this application of sulphation may be found in Histological and Histochemical Methods: Theory and Practice, Chapter 10 , page 143, of the 2nd ed. (Kiernan, 1990, Pergamon Press, Oxford) but this has been out of print for about a year. It will also be in Chapter 10 in the 3rd ed. (Butterworth-Heinemann, Oxford, in press, due out early 1999).

John A. Kiernan, The University of Western Ontario

\title{
A Tip on Scanning TEM Negatives with Flatbed Scanners
}

Many 10 and 12-bit per channel flatbed scanners offer a transparency adapter for scanning film negatives. However, scanning TEM negatives in the negative transparency mode - the obvious thing to do - often yields horrible results that might make you think the scanner is no good. The problem arises because the negative transparency scanning mode is intended for low-contrast photographic negative film, not the high-contrast film used in TEM. Scanner driver programs typically contain built-in contrast compensation (a kind of gamma correction) for photographic nega-

\section{How To Always Have Fresh $\mathrm{H}_{2} \mathrm{O}_{2}$ Solutions on Hand}

Hydrogen peroxide solutions eventually deteriorate in storage, shelf life being shorter the higher the percentage of $\mathrm{H}_{2} \mathrm{O}_{2}$. This can happen even more quickly if there is accidental contamination of the stock solution (e.g., by metal). I have had this happen twice, and think the cause was using a syringe and needle to withdraw a small volume of $30 \% \mathrm{H}_{2} \mathrm{O}_{2}$ for dilution. A week later, the $30 \% \mathrm{H}_{2} \mathrm{O}_{2}$ was $100 \% \mathrm{H}_{2}$ )!

Urea hydrogen peroxide is a stable, solid alternative. It is in the Sigma catalog: \#U1753 (powder) and U8879 (tablets, each containing $1.75 \mathrm{mg}$ of $\mathrm{H}_{2} \mathrm{O}_{2}$ ). It is a clathrate, with urea molecules making a cage round the $\mathrm{H}_{2} \mathrm{O}_{2}$.

Weigh it out as if it were $35 \% \mathrm{H}_{2} \mathrm{O}_{2}$, and make a stock solution ( $1 \% \mathrm{wt}$ vol in water is convenient) for further dilution. Use $1 / 35 \mathrm{~g}=28.6 \mathrm{mg}$ for 100 $\mathrm{mL}$ of $1 \% \mathrm{H}_{2} \mathrm{O}$.

This stock $1 \%$ solution can be replaced every day or two, so it's always fresh.

John A. Kiernan, The University of Western Ontario, London, Canada

\section{A Recipe For Eosin Plus Phloxine For Hematoxylin-Eosin Staining}

Eosin has been the traditional counterstain for hematoxylin when staining tissue for light microscopy. A synthetic dye, eosin has an affinity for acidophilic components in cells ${ }^{1}$. There are many different formulae for eosin depending on the intensity of staining of tissue components desired. Eosin $Y$ gives a pinkyellow coloration: eosin $\mathrm{B}$, pink-blue: and picric acid* can be added to intensify connective tissue staining. Eosin $Y$ is the dye most commonly used in formulations today.

Eosin is $\mathrm{pH}$ dependent. At $\mathrm{pH} 4.5$ - 5, eosin stains erythrocytes and keratin red/orange; collagen, pink; and smooth muscle, light pink. With the addition of phloxine $B$, collagen stains bright pink-rose in contrast to the pink smooth muscle.

This laboratory has used both commercially prepared and laboratory prepared eosin solutions. The directions for the laboratory-prepared solution ${ }^{3}$ are as follows:

Eosin $Y(C . I .45380) \quad 1.0 \mathrm{gm}$

Phioxine B (C.I. 45410) $0.1 \mathrm{gm}$ 